\title{
Visualization of spatiotemporal changes between DEMs via Morphological Interpolation
}

\author{
Sin Liang Lim ${ }^{1}$, Sim Hui Tee ${ }^{2}$, Sin Ting Lim $^{3}$
}

\begin{abstract}
Digital Elevation Model (DEM) is a digital model representation of the surface of a terrain. It consists of elevation values sampled at regular intervals which could be used to study vast types of phenomena across spatial positions. However, acquisition of DEM data in continuous sequences at very high spatial resolution is very costly from the point of cost, manpower, and storage requirement. In this paper, we propose a method to visualize the morphological changes for a same area over a period using two DEM images. Through mathematical morphology approach, the interpolated sequences between these pair of DEMs are hierarchically generated. These set of hierarchically interpolated medians paves a way to visualize the spatiotemporal changes which have happened in a particular area at discrete-time intervals. The potential application which we could implement the proposed framework include natural disaster surveillance and monitoring (eg. Landslide, flood, earthquake).
\end{abstract}

Keywords - digital elevation model (DEM), mathematical morphology, morphological interpolations

\section{Introduction}

With the rapid growth and development of high resolution remotely-sensed images, surveillance and detection of land and/or river changes has been a topic of interest. The observation of changes in land could be used to monitor the natural disaster such as landslide or earthquake, while the study of river basin changes could assist us in flood mitigation. If we were able to visualize the changes in Earth surface in both spatial and timely manner across different time, we will have a better understanding on the interactions and relationships between human and natural phenomena [1]. This will further help us to better manage and utilize the available resources.

Digital Elevation Model (DEM) is a raster representation of a terrain surface in 3-dimention [2]. Each point in DEM data denotes the elevation matrix for each ground point in certain cell size. DEM is widely used in many aspects of social development and economic construction, including land-use planning, environmental management, disaster monitoring and relief, and national security defense [3]. The DEM could be acquired through techniques such as photogrammetry, LiDAR, SAR, land surveying, etc. [4]. DEMs are often used in geographic information systems, and serves as the basis for digitally produced relief maps. Applications where DEM are most commonly applied include landscape modeling, city planning, flood or drainage modeling, land-use studies, and other geological applications [5].

Faculty of Engineering, Multimedia University ${ }^{1}$, Faculty of Creative Multimedia $^{2}$, Faculty of Engineering and Technology ${ }^{3}$ Malaysia
Many interpolation techniques such as Inverse Distance Weighting (IDW), Kriging, and Spline are commonly applied in constructing DEM at different scales (eg. [6]). However, we will not address the interpolation techniques to generate DEMs at field scale, but we propose the visualization method to view the continuous sequence from the changes for a specific area of interest over a time period. The hierarchically generated morphing-like sequence at discrete time intervals between the pair of DEMs would enable us to study the spatiotemporal changes which happen from one year to succeeding years. It is noted that we implement our proposed method on grayscale DEM images instead of binary images [eg. 7].

Mathematical morphology, a concept based on set theory, is proven to be robust and successful in processing and analyzing digital images [8]. The basic morphology operators are erosion and dilation, where the combination of these operators form the extension of the other operators, including spatial interpolation [9]. In this paper, we follow the framework as proposed in our previous work $[10,11]$ on a pair of DEMs of a same region but acquired in two different years. By doing so, it will be interesting to visualize the interpolated sequences constructed over years. Particularly, the visualization of changes across time will help us to monitor the land-use in the context of our study area, in this case, Kuala Lumpur--the capital of Malaysia. It is interesting to study the changes in the city due to urbanization and development.

\section{Grayscale Morphological Operations}

\section{A. Grayscale Morphological Operations}

Let $f(x, y)$ represents a grayscale digital image containing discrete values at each $(x, y)$ position. It is assumed that there are $(\mathrm{Z}+1)$ possible intensity values where $m=0,1,2, \ldots, M$. Since the image is denoted as gray level, therefore each pixel is assigned with 8 bit which results in $M=255$.

The two fundamental operators of morphological operations are dilation and erosion. With the use of a structuring element (symmetric about origin, flat and rhombic, with primitive size of $3 \times 3$ ) shown in Figure 1 b, the grayscale image in Figure 1a is eroded into Figure 1c and dilated into Figure 1d, respectively. The $\Theta$ and $\Theta$ are symbols used to represent dilation and erosion, and their mathematical transformations are defined as follow [12]: 
Proc. of the Sixth Intl. Conf. Advances in Computing, Communication and Information Technology- CCIT 2018 Copyright (C) Institute of Research Engineers and Doctors, USA. All rights reserved.

ISBN: 978-1-63248-149-8 doi: 10.15224/978-1-63248-149-8-20

$$
\begin{aligned}
& (f \oplus B)(x, y)=\max _{(i, j) \in B}\{(x-i, y-j)\} \\
& (f \Theta B)(x, y)=\min _{(i, j) \in B}\{(x+i, y+j)\}
\end{aligned}
$$

\begin{tabular}{|c|c|c|c|c|c|c|c|c|c|}
\hline 92 & 183 & 250 & 200 & 191 & & & & & \\
\hline 160 & 145 & 89 & 68 & 54 & & 0 & 1 & 0 & \\
\hline 32 & 70 & 120 & 53 & 136 & & 1 & 1 & 1 & \\
\hline 9 & 101 & 44 & 60 & 98 & & 0 & 1 & 0 & \\
\hline 122 & 83 & 72 & 125 & 5 & & & & & \\
\hline \multicolumn{5}{|c|}{ (a) } & \multicolumn{5}{|c|}{ (b) } \\
\hline 183 & 250 & 250 & 250 & 200 & 92 & 92 & 89 & 68 & 54 \\
\hline 160 & 183 & 250 & 200 & 191 & 32 & 70 & 68 & 53 & 54 \\
\hline 160 & 145 & 120 & 136 & 136 & 9 & 32 & 44 & 53 & 53 \\
\hline 122 & 101 & 120 & 125 & 136 & 9 & 9 & 44 & 44 & 5 \\
\hline 122 & 122 & 125 & 125 & 125 & 9 & 72 & 44 & 5 & 5 \\
\hline \multicolumn{5}{|c|}{ (c) } & \multicolumn{5}{|c|}{ (d) } \\
\hline 92 & 92 & 92 & 89 & 68 & 160 & 183 & 250 & 200 & 191 \\
\hline 92 & 92 & 89 & 68 & 54 & 160 & 145 & 120 & 136 & 136 \\
\hline 32 & 70 & 68 & 53 & 54 & 122 & 101 & 120 & 120 & 136 \\
\hline 9 & 72 & 44 & 53 & 53 & 101 & 101 & 101 & 120 & 125 \\
\hline 72 & 72 & 72 & 44 & 5 & 122 & 101 & 120 & 125 & 125 \\
\hline \multicolumn{5}{|c|}{ (e) } & \multicolumn{5}{|c|}{ (f) } \\
\hline
\end{tabular}

Figure 1. (a) Grayscale image, $f(x, y)$, (b) Structuring element $B$ of primitive size $3 \times 3$, (c) Dilation, (d) Erosion, (e) Opening, and (f) Closing of $f(x, y)$ with respect to $B$. [11]

From Figure 1, it is obvious that erosion takes the central values as the minimum gray values within the neighborhood window image, while dilation fills the central values with the maximum gray values in the neighborhood. As such, erosion darkens the original image while dilation brightens the image.

Furthermore, the concatenation of dilation and erosion results in two other morphology operators, namely opening and closing. The equations for opening and closing operations are given as:

$$
\begin{aligned}
& (f \circ B)(x, y)=(f \Theta B) \oplus B \\
& (f \bullet B)(x, y)=(f \oplus B) \Theta B
\end{aligned}
$$

The symbols $\circ$ and $\bullet$ are used to represent opening and closing, separately. Opening removes particular image details which are smaller than structuring element $B$, whereas closing fills holes in objects and joins small breaks in the image.

\section{B. Multiscale Grayscale Morphological operators}

Iterative morphological operations can be generated by controlling the size of the structuring element B. This transformation is known as multiscale operations, where the size of $n B$ is increased as $n=0,1,2,3, \ldots, N$. For example, multiscale dilation $(f \oplus n B)$ is generated as:

$$
(f \oplus n B)=(f \oplus B) \oplus B \oplus B \ldots \oplus B
$$

Similarly, multiscale erosion, opening, and closing can be expressed as:

$$
\begin{aligned}
& (f \Theta n B)=(f \Theta B) \Theta B \Theta B \ldots \Theta B \\
& (f \circ n B)=(f \circ B) \circ B \circ B \ldots \circ B \\
& (f \bullet n B)=(f \bullet B) \bullet B \bullet B \ldots \bullet B
\end{aligned}
$$

at scale $n=0,1,2,3, \ldots, N$.

\section{Infima and Suprema}

Let $f$ and $g$ represent two DEMs in grayscale, and let infima $(f \wedge g)$ and suprema $(f \vee g)$ of these DEMs be represented as:

$$
\begin{aligned}
& (f \wedge g)=\inf (f, g)=\min \{f(x, y), g(x, y)\} \\
& (f \vee g)=\sup (f, g)=\max \{f(x, y), g(x, y)\}
\end{aligned}
$$

\section{Morphological Median}

Based on [10], the morphological median between $f$ and $g$, denoted as $M(f, g)$, can be produced as:

$$
M(f, g)=\stackrel{N}{\vee} \underset{n=0}{\vee}\left(M_{n}(f, g)\right)
$$

where

$$
M_{n}(f, g)=((f \wedge g) \oplus n B) \wedge((f \vee g) \Theta n B)
$$

Morphological median will then be hierarchically generated to obtain the interpolated sequences between $f$ and $g$ [13].

\section{DEM Data and Study Area}

The study area is chosen as a region in Kuala Lumpur, the national city of Malaysia. It is the largest city in the country with an estimated population of 1.79 million of as 2017 [14]. Besides being the cultural, financial and economic centre of Malaysia, Kuala Lumpur is among the fastest growing metropolitan regions in South East Asia. 
The geography of Kuala Lumpur is mainly influenced by the huge Klang Valley. It is bordered by the Titiwangsa Mountains in the east, several minor ranges in the north and the south and the Strait of Malacca in the west. The name "Kuala Lumpur" rooted from a Malay term which means "muddy confluence" as it is located at the confluence of the Klang and Gombak rivers [15]. The city covers an area of 243 $\mathrm{km}^{2}$ [16] with an average elevation of $81.95 \mathrm{~m}$ [17].

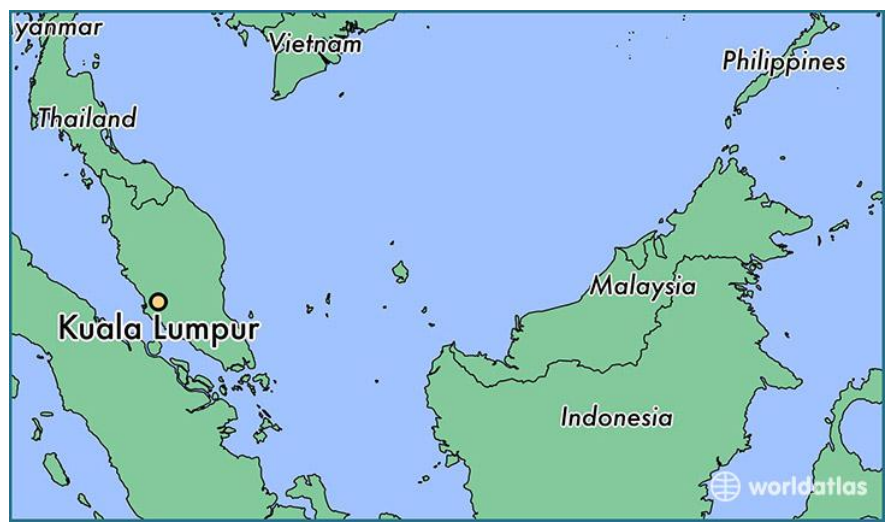

Figure 2. Location of Kuala Lumpur [18]

Unfortunately, flood is a frequent occurrence in Kuala Lumpur especially after heavy downpours due to lack of structural irrigation following the rapid development within the city centre [19]. As such, in this paper, we propose a visualization method to view the changes of a study area in Kuala Lumpur which occur over years in hope to help the experts to study the causes and further prevent/mitigate natural disaster such as flood.

We obtained a pair of DEM data of a same region in Kuala Lumpur from United States Geological Survey (USGS) EarthExplorer website (https://earthexplorer.usgs.gov/), with a time gap of 11 years. The first DEM data was acquired from Shuttle Radar Topography Mission (SRTM) on 11 February 2000, while the second DEM data was acquired from Advanced Spaceborne Thermal Emission and Reflection Radiometer (ASTER) Global Digital Elevation Model Version 2 (GDEM V2) on 17 October 2011. The study region encompasses the center coordinate of $4^{\circ} \mathrm{N}, 101^{\circ} \mathrm{E}$. Both DEMs have the resolution of 30 meters with original size of $3601 \times$ 3601 pixels (Figures $3 a$ and $3 b$ ). However, in order to ease the computation, smaller areas of $512 \times 512$ pixels are taken for both DEMs, as shown in Figures $3 \mathrm{c}$ and $3 \mathrm{~d}$.
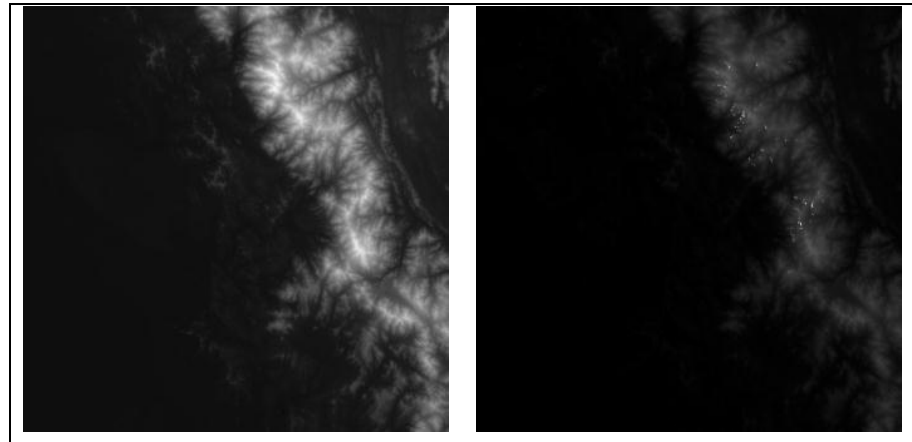

(a)

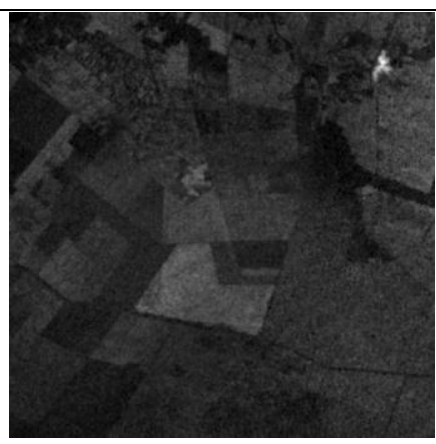

(c) (b)

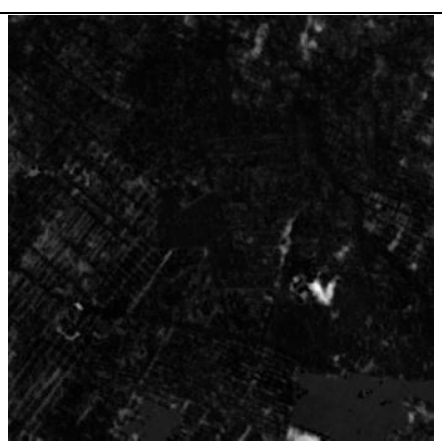

(d)
Figure 3. DEMs of the study area in Kuala Lumpur in two different years. (a) Original area from SRTM, (b) Original area from ASTER GDEM, (c) Sub area from SRTM, and (d) Sub area from ASTER GDEM. All DEM data are courtesy of the U. S. Geological Survey.

\section{Results and Discussion}

For discussion purpose, four levels of successive morphological medians are generated between DEM1 (ie. Figure 3c) and DEM2 (ie. Figure 3d) using flat disk structuring element (Figure 1b) with primitive size of $3 \times 3$. As a result, a total of 15 morphological medians are generated. These include one at $0^{\text {th }}$-level, two at $1^{\text {st }}$-level, four at $2^{\text {nd }}$ level, and eight at $3^{\text {rd }}$-level. The morphological interpolations obtained for a study region in Kuala Lumpur with a time difference of 11 years are shown sequentially in Figure 4.

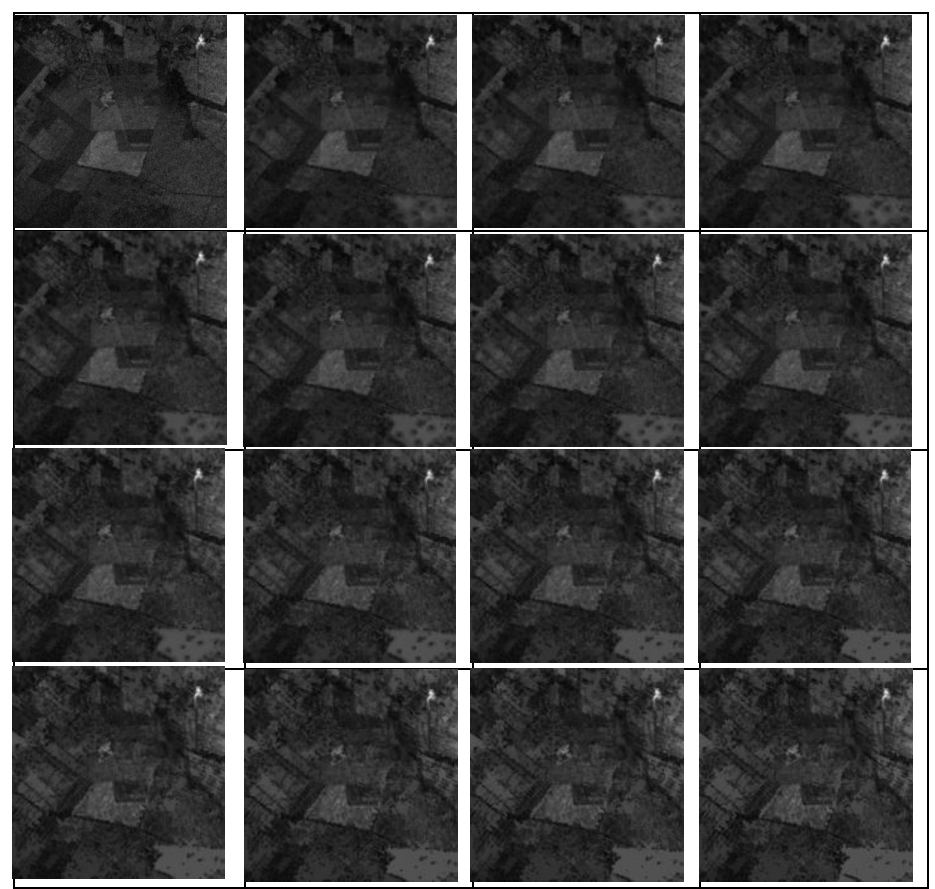


Proc. of the Sixth Intl. Conf. Advances in Computing, Communication and Information Technology- CCIT 2018

Copyright (C) Institute of Research Engineers and Doctors, USA. All rights reserved.

ISBN: 978-1-63248-149-8 doi: 10.15224/978-1-63248-149-8-20
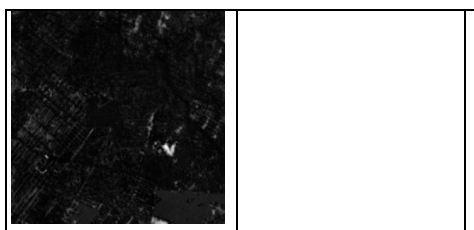

Figure 4. Grayscale morphological interpolations generated for a study region in Kuala Lumpur across a period of 11 years.

Figure 4 shows the series of interpolated images generated for a study region in Kuala Lumpur over a period of 11 years. Although the variances are not significantly noticeable from one interpolated image to another, it is undoubtedly showing changes spatially. This can be proven in Figure 5 from the graph of area plotted for each interpolated image.

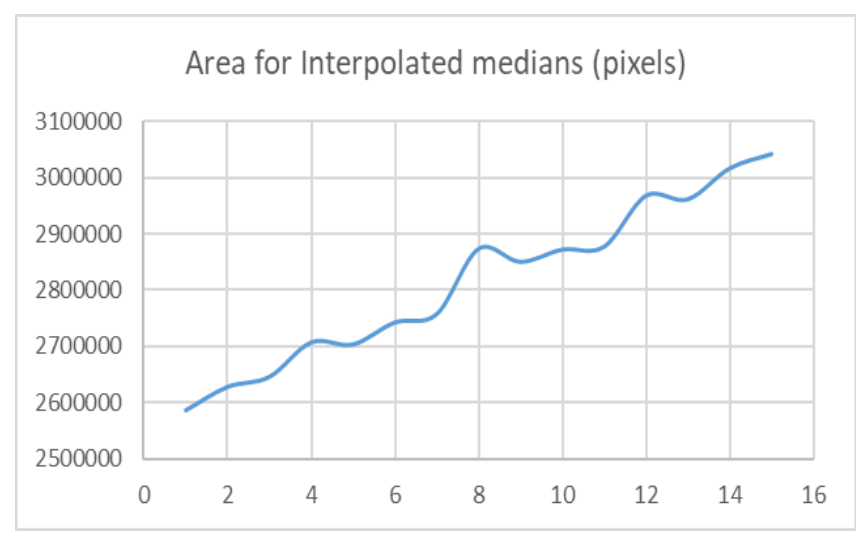

Figure 5. Graph showing the area of interpolated images in pixels.

In addition, based on the results shown in Figure 4, we are certain that the morphologically generated interpolations between SRTM DEM and ASTER GDEM produce smooth transition from one DEM to another. It is interesting to note each morphological median takes few number of iterations to reach the convergence level, as shown in Figure 6:

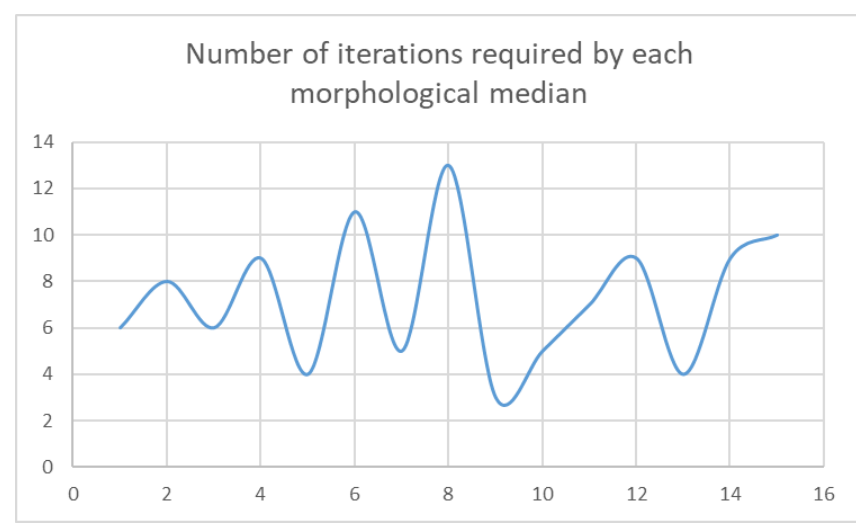

Figure 6 Number of iterations required to generate 15 morphological medians hierarchically between a study region in Kuala Lumpur with a time difference of 11 years.

\section{v. Conclusion}

In this paper, we tested our proposed framework on a pair of DEM data for a region in Kuala Lumpur, Malaysia. We are interested to visualize the spatiotemporal changes which take place across time from these two DEM data. The first DEM data was acquired from SRTM in year 2000 while the second DEM data was acquired from ASTER in year 2011. Using our proposed method, the sequential set of morphologically interpolated DEMs are hierarchically computed across discrete time-steps. It is observed that the metamorphs from one DEM to another DEM across time is rather smooth. Besides, the interpolation process requires less number of iterations to achieve convergence level. As such, it is concluded that the proposed method in this paper serves as a good alternative to view and subsequently analyze the changes which occur for an area over a time period.

\section{Acknowledgment}

The authors are thankful to the Ministry of Higher Education Malaysia for the award of Fundamental Research Grant Scheme FRGS/1/2015/TK04/MMU/02/10. Besides, we are also grateful to U.S. Geological Survey (USGS) for allowing free access to DEM data in their EarthExplorer website (https://earthexplorer.usgs.gov/).

\section{References}

[1] L. Deren, X. Song, J. Wanshou, S. Zhenfeng, "An efficient approach for detecting changes of terrain and updating old DEM", International Society for Photogrammery and Remote Sensing, Symposium of ISPRS Commission IV, Volume XXXVI Part 4, 2005 (5 pages).

[2] M. S. S. K Dr. Pratibha P. Shingare 1, "Review on Digital Elevation Model," Internatinoal Journal of Modern Engineering Research (IJMER), vol. 4, no. 4, Jul - Aug, pp. 2412 - 2418, 2013.

[3] L. Jian-jun, W. Dong-hua, S. Yao-ling, Z. Wen-hao, K. Xi, "Updating and refinment of national 1:500000 DEM", International Society for Photogrammery and Remote Sensing, International Workshop of Geopatial Information, pp. 114-122, 2013.

[4] L. Junhua and C. Wenjun, "A rule-based method for mapping Canada's wetlands using optical, radar, and DEM data", International Journal of Remote Sensing, vol. 26, issue 22, pp. 5051-5069, 2005.

[5] I. Balenovic, H. Marjanovic, D. Vuletic, et. al., "Quality assessment of high density digital surface model over different land cover classes", Periodicum Biologorum, vol. 117, no 4, pp. 459-470, 2015.

[6] P. Arun, "A comparative analysis of different DEM", The Egyption Journal of Remote Sensin and Space Sciences, vol. 16, pp. 133-139, 2013.

[7] J. Serra, "Interpolations et distance de Hausdorff", Tech. Rep N15/94/MM, Ecole des Mines de Paris, 1994.

[8] J. Serra, "Image analysis and mathematical morphology", London, U.K.: Academic, 1982.

[9] J. Serra, "Hausdorff distances and interpolations", in Mathematical morphology and its applications to images and signal processing (Eds. Henk. J. A. M. Heijmans, and Jos B. T. M. Roerdink), Dordrecht, The Netherlands, Kluwer Academics Publishers, 1998.

[10] B. S. D. Sagar and S. L Lim, "Morphing of grayscale DEMs via morphological interpolations", IEEE Journal of Selected Topics in Applied Earth Observation and Remote Sensing, vol. 8, no. 11, pp. 5190-5198, 2015

[11] S. L Lim, S. H. Tee, and S. T. Lim, "Morphological interpolations of low-resolution DEMs vs High-resolution DEMs: A comparative study", Progress in Computer Sciences and Information Technology International Conference, Procsit 20-22 December 2016.

[12] S. R. Sternberg, "Grayscale morphology", Computer Visual, Graphics, and Image Processing, vol. 35, pp. 333-355, 1986. 
[13] M. Iwanowsky, "Application of Mathematical Morphology to Image Interpolation", $\mathrm{PhD}$ thesis, School of Mines Paris-Warsaw University of Technology, 2000.

[14] "Federal Territory of Kuala Lumpur". Department of Statistics, Malaysia. Retrieved 5 March 2018.

[15] Kuala Lumpur: Growing Pains", Asia's Best Cities 2000, Asiaweek. Retrieved 5 March 2018.

[16] Laporan Kiraan Permulaan 2010", Jabatan Perangkaan Malaysia. pp. 27. Retrieved 5 March 2018.

[17] "Kuala Lumpur Location", Malaysia Travel. Retrieved 5 March 2018.

[18] https://www.worldatlas.com/as/my/14/where-is-kuala-lumpur.html. Retrieved 5 March 2018.

[19] "Kuala Lumpur Environment", Kuala Lumpur City Hall. Retrieved 5 March 2018.

About Authors:

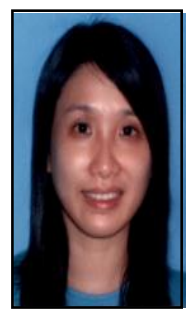

Dr. Lim Sin Liang is a senior lecturer from Faculty of Engineering, Multimedia University. Her research interests include terrestrial pattern retrieval using mathematical morphology, convexity measure and geodesic spectrum in digital topographic basins.

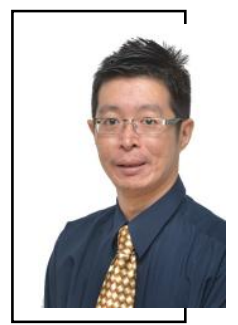

Dr. Tee Sim Hui is a senior lecturer from Faculty of Creative Multimedia, Multimedia University. His research interest includes bioinformatics and software engineering.

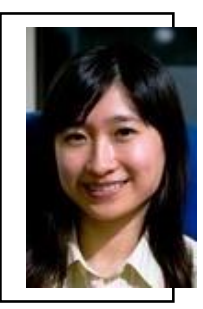

Lim Sin Ting is a lecturer from Faculty of Engineering and Technology in Multimedia University. Her research area includes region-based medical image compression and medical image instrumentation particularly on $\mathrm{CT}$ and MRI scanners. 